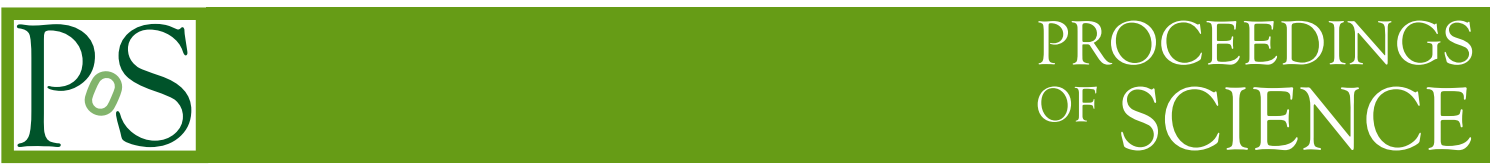

\title{
Dilepton Production in Heavy-Ion Collisions
}

\author{
Ralf Rapp* \\ Department of Phyiscs \& Astronomy and Cyclotron Institute, Texas A\&M University, College \\ Station, TX 77843 \\ E-mail: rapp@comp.tamu.edu
}

\begin{abstract}
The properties of electromagnetic radiation from hot fireballs as created in ultra-relativistic heavyion collisions are reviewed. We first outline how the medium effects in the electromagnetic spectral function, which governs thermal production rates, relate to the (partial) restoration of chiral symmetry. In particular, we show how chiral and QCD sum rules, together with constraints from lattice QCD, can render these relations quantitative. Turning to dilepton data, we elaborate on updates in the space-time evolution and quark-gluon plasma emission rates from lattice-QCD calculations. With a now available excitation function in dilepton spectra from the RHIC beam-energy scan connecting down to SPS energies, we argue that a consistent interpretation of dilepton data emerges. Combining well-constrained space-time evolutions with state-of-the-art emission rates identifies most of the radiation to emanate from around the pseudo-critical temperature, and thus confirms resonance melting as the prevalent mechanism in this regime, compatible with chiral restoration. Recent measurements of a relatively soft slope and large elliptic flow in direct-photon spectra at RHIC and LHC lend further support to this picture.
\end{abstract}

8th International Workshop on Critical Point and Onset of Deconfinement,

March 11 to 15, 2013

Napa, California, USA

* Speaker. 


\section{Introduction}

Electromagnetic (EM) radiation off the expanding medium created in energetic collision of heavy nuclei may provide a pristine glimpse at the hot QCD matter formed in these reactions. However, the measured spectra constitute, radiation yields integrated over the entire lifetime of the fireball. To effectively discriminate the different components in the spectra (e.g., primordial production, early QGP radiation, hot/dense matter around $T_{c}$ and late hadronic emission), the full richness of this observable needs to be exploited. In this regard, and advantageous feature of the local thermal emission rate,

$$
\frac{d N_{l l}}{d^{4} x d^{4} q}=\frac{\alpha_{\mathrm{EM}}^{2} L(M)}{6 \pi^{3} M^{2}} f^{B}\left(q_{0} ; T\right) \rho_{\mathrm{EM}}\left(M, q ; \mu_{B}, T\right),
$$

is its separate dependence on the invariant mass $(M)$ and 3-momentum $(q)$ through the vector spectral function, $\rho_{\mathrm{EM}}$, of the medium. On the one hand, the $M$-dependence encodes the dynamical effects of the microscopic interactions governing its shape (e.g., information on the degrees of freedom or chiral symmetry restoration). On the other hand, the momentum dependence mostly probes the kinematics of the medium, i.e., the interplay of decreasing temperature and increasing blue shift in the fireball expansion, well-known from hadron spectra at thermal freeze-out. For EM radiation, this interplay is encoded in a superposition of all phases of the fireball, and thus requires further disentangling. The main lever arm here is the competition between the Bose factor, $f^{B}$, favoring early phases, and the emitting 3 -volume, $V_{\mathrm{FB}}$, favoring late phases. Since the temperature sensitivity of the Bose factor increases exponentially at large energies (i.e., large mass, large 3-momentum, or both), the latter prevail at high temperatures, while the weaker sensitivity at small $q_{0}$ shifts the main emission to smaller temperatures where the radiating volume grows with an inverse power in $T$. At a quantitative level, the temperature/density dependence of the spectral function also figures into these considerations, especially in the low-mass regime (LMR, $M \leq 1 \mathrm{GeV}$ ). Another valuable diagnostic tool that has recently become available in the EM sector is the elliptic flow. Since for EM spectra the $v_{2}$ is a weighted sum over all phases, its magnitude, relative to the final-state hadrons, can serve as another indicator of the emission time; in typical hydrodynamic evolutions at RHIC, the bulk elliptic flow takes about $5 \mathrm{fm} / c$ to develop most of its strength. Clearly, robust interpretations of the spectral shape of the emitted radiation need to be in concert with a good control over the emission temperatures.

In the following, we will first give an update on implications of hadronic medium effects in the vector spectral function for chiral restoration and introduce Quark-Gluon Plasma (QGP) emission rates motivated by thermal lattice-QCD (1QCD) computations (Sec. 2). This also raises the issue of consistency of microscopic emission rates and the equation of state $(\mathrm{EoS})$ governing the bulk evolution, in particular the local temperature. We will then turn to a discussion of low-mass dilepton spectra from SPS to top RHIC energy, and address recent measurements of spectra and elliptic flow of direct photons at RHIC and LHC (Sec. 3). A brief conclusion is given in Sec. 4.

\section{Thermal EM Emission Rates and Chiral Symmetry Restoration}

Effective hadronic Lagrangians, combining chiral pion interactions with resonance excitations, implemented into finite-temperature field theory, have been widely applied to evaluate vector- 

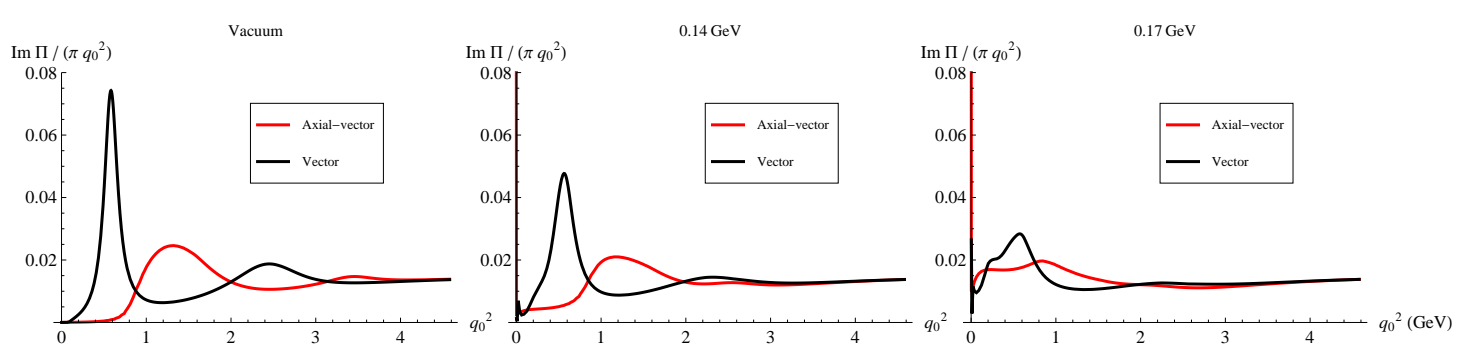

Figure 1: Isovector-vector and -axialvector spectral functions in vacuum (left panel) and at finite temperature (middle panel: $T=140 \mathrm{MeV}$, right panel: $T=170 \mathrm{MeV}$ ) [3].

meson spectral functions in hot and/or dense hadronic matter, see, e.g., Ref. [1, 2] for recent reviews. The generic outcome of these calculations is an appreciable broadening of the spectral peaks with little, if any, mass shift, provided that the parameters of the vacuum Lagrangian (coupling constants and bare masses) are not subject to in-medium changes. For the $\rho$ meson, the broadening amounts to a few hundred $\mathrm{MeV}$ at hadronic densities of $\rho_{h}=0.2 \mathrm{fm}^{-3}$, leading to its melting when extrapolated into the regime of the expected QCD phase boundary $\left(T_{\mathrm{pc}} \simeq 170 \mathrm{MeV}\right)$, cf. the black lines in Fig. 1. The dissolution of the hadronic resonance structure suggests a change of the relevant degrees of freedom in the system, and thus may be interpreted as an indicator of deconfinement [4]. Another issue is if and how these medium effects signal the restoration of the spontaneously broken chiral symmetry. This is quantified by Weinberg sum rules (WSRs) [5, 6],

$$
f_{n}=-\int_{0}^{\infty} d s s^{n}\left[\rho_{V}(s)-\rho_{A}(s)\right],
$$

which relate moments of the difference between the isovector-vector and -axialvector spectral function to order parameters of chiral breaking, $f_{-2}=f_{\pi}^{2}\left\langle r_{\pi}^{2}\right\rangle / 3-F_{A}, f_{-1}=f_{\pi}^{2}, f_{0}=f_{\pi}^{2} m_{\pi}^{2}$, $f_{1}=-2 \pi \alpha_{s}\left\langle\mathscr{O}_{4}^{\chi}\right\rangle\left(r_{\pi}\right.$ : pion charge radius, $F_{A}$ : coupling constant for the radiative pion decay, $\left\langle\mathscr{O}_{4}^{\chi}\right\rangle$ : chirally breaking 4-quark condensate). These sum rules remain valid at finite temperature [7], independently at each 3-momentum for longitudinal and transverse components. Ideally, one would compute both in-medium spectral function in a chiral approach, evaluate the integrals and test for agreement with the order parameters, as given, e.g., by thermal 1QCD. However, realistic calculations of the in-medium axialvector spectral function are not yet available. In Ref. [3], a more modest question has been addressed, namely whether solutions to Weinberg and QCD sum rules, with order parameters from IQCD as available, can be found using existing in-medium calculations of the $\rho$ spectral function [8]. Toward this end, a quantitative fit to the axial-/vector $\tau$ data was employed which accurately satisfies the sum rules in vacuum. In this fit, Breit-Wigner ansätze for the $a_{1}$ and excited resonances ( $\rho^{\prime}$ and $a_{1}^{\prime}$ ) have been used, and their masses and widths were required to change monotonically with temperature. A viable solution was indeed found, with the resulting spectral functions clearly exhibiting their mutual approach toward degeneracy, see Fig. 1. While this is not a proof of chiral restoration, it nevertheless shows that a strongly broadened $\rho$ spectral function, as will be used in applications to dilepton data below, is compatible with it. Another indication for this compatibility arises from the realization that the processes generating the $\rho$ broadening (resonances and pion cloud modifications) find their counterparts in reducing the chiral 

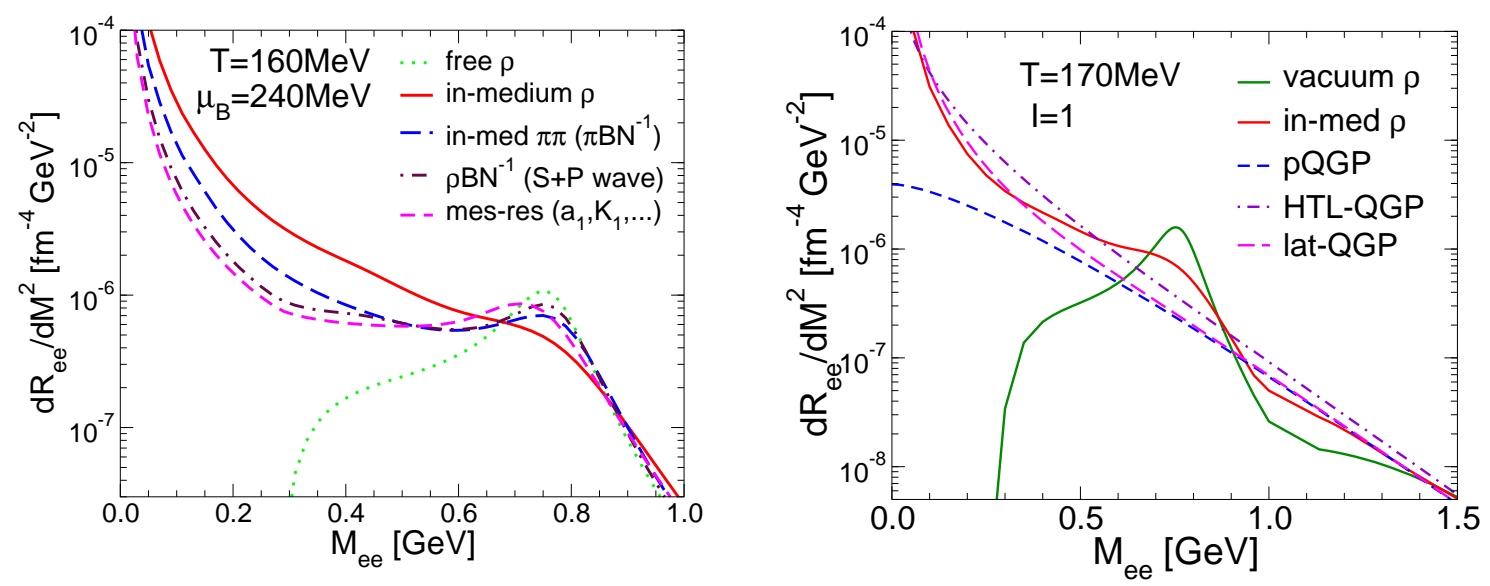

Figure 2: Thermal dilepton rates (integrated over 3-momentum) in hot/dense matter characteristic for top SPS energy (left panel) and top RHIC energy (right panel). The former shows the total hadronic rate and its decomposition into pion-cloud and resonance channels while the latter compares the total vacuum and in-medium hadronic rates to QGP emission within the hard-thermal-loop approach [12] and IQCD [13].

condensate. In dilute matter, the latter decreases according to [9]

$$
\frac{\langle\bar{q} q\rangle\left(T, \mu_{B}\right)}{\langle\bar{q} q\rangle_{0}}=1-\sum_{h} \frac{\rho_{h}^{s} \Sigma_{h}}{m_{\pi}^{2} f_{\pi}^{2}}
$$

( $\rho_{h}^{s}$ : scalar density), where $\Sigma_{h}=m_{q}\langle h|\bar{q} q| h\rangle$ is characterized by the scalar quark number inside hadron $h$; it can be decomposed into contributions from its quark core and pion cloud [10,11], $\Sigma_{h}=\Sigma_{h}^{\text {core }}+\Sigma_{h}^{\pi}$, which are roughly equal in magnitude (in analogy to the medium effects in the dilepton rate, see left panel of Fig. 2). The resulting suppression of the quark condensate from a (non-interacting) hadron resonance gas reproduces lQCD calculations rather well [14].

Progress has been made in extracting the QGP emission rate from IQCD at zero pair momentum, $q=0[13,15]$. Applications to experiment require the extension to finite $q$; in Ref. [16] this has been constructed by implementing the $q=q_{0}$ dependence of the perturbative photon rate and matching it to the conductivity in IQCD. The $q$-integrated rates are similar in shape to the hardthermal-loop results, albeit quantitatively somewhat smaller in the LMR. One reason for this could be the smaller number of degrees of freedom that characterize the (nonperturbative) QGP in 1QCD relative to the perturbative system underlying the HTL rates. It is thus important to maintain consistency between emission rates and EoS in applications to heavy-ion collisions (as will be done below). One also sees from the right panel of Fig. 2 that, for temperatures around $T=170 \mathrm{MeV}$, the bottom-up extrapolated in-medium hadronic rates approximately coincide with the top-down extrapolated QGP rates.

Microscopic calculations of in-medium dilepton rates can be straightforwardly carried to the photon point, i.e., $M=0$ and $q_{0}=q$. For hadronic rates this has been done in Ref. [17], where, in addition, $\omega t$-channel exchange in $\pi \rho \rightarrow \pi \gamma$ has been identified as an important photon source at $q_{t} \gtrsim 1.5 \mathrm{GeV}$. It turns out that the hadronic emission rate is quite comparable to the complete leading-order QGP rate [18] in the vicinity of $T_{\mathrm{pc}}$. 

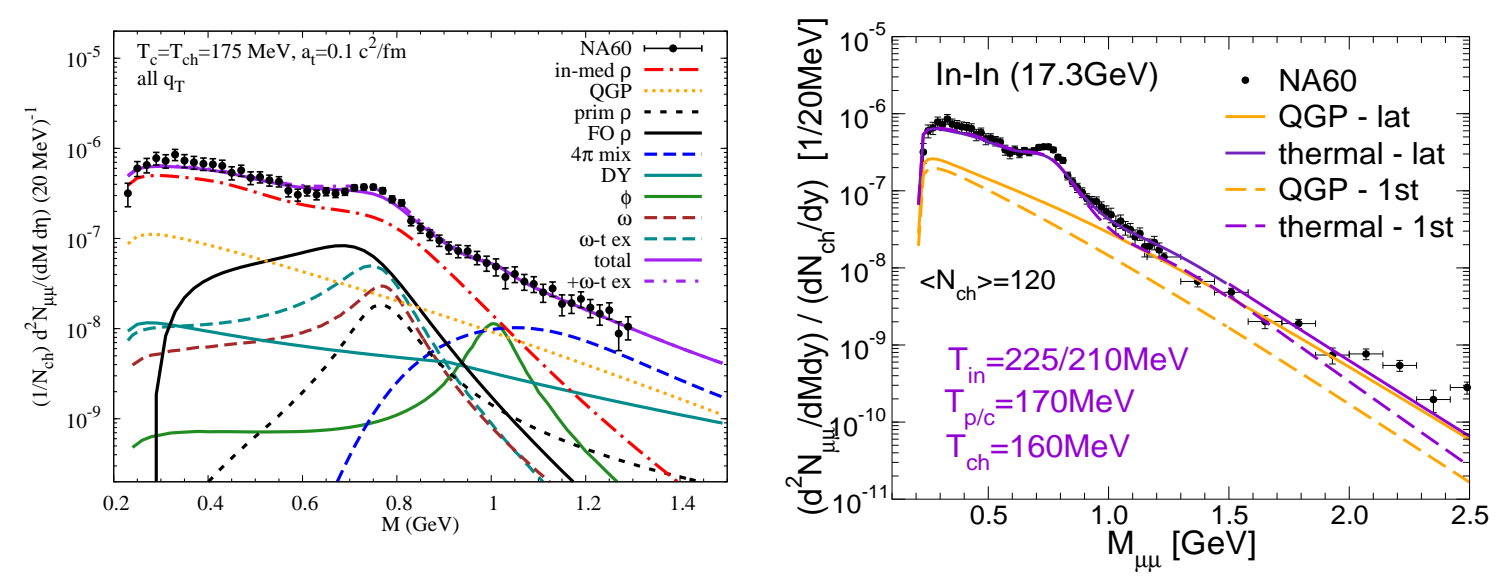

Figure 3: Dimuon excess spectra in $\operatorname{In}-\operatorname{In}(\sqrt{s}=17.3 \mathrm{GeV})$ collisions at SPS as measured by NA60 (acceptance corrected) [19], compared to theoretical calculations with in-medium vector spectral functions (left panel) [1], and illustrating their sensitivity to the QGP EoS (right panel).

\section{EM Spectra in Heavy-Ion Collisions}

In the following we confront the models of in-medium hadronic and QGP rates (as discussed above), after folding over thermal fireballs constrained by hadron data (yields, spectra and $v_{2}$ ), to dilepton (Sec. 3.1) and photon data (Sec. 3.2) at SPS, RHIC and LHC.

\subsection{Low-Mass Dileptons}

In the NA60 dimuon excess spectra [19] the contributions from final-state hadron decays could be subtracted thanks to excellent statistics and mass resolution. In the LMR, the predictions of a melting $\rho$ resonance agree well with the data, while continuum radiation from multi-hadron annihilation and the QGP figures for $M>1 \mathrm{GeV}$ (Fig. 3 left). The relative contributions and spectral shape (as given by the in-medium spectral function and overall Bose factor) in the invariant-mass spectra are determined by the temperature profile of the fireball, cooling from $T_{i} \simeq 200 \mathrm{MeV}$ to $T_{\mathrm{fo}} \simeq 120 \mathrm{MeV}$, with little sensitivity to the expansion dynamics. The overall yield could not be accurately predicted, but rather allows to extract the (average) fireball lifetime as $\tau_{\mathrm{FB}}=6.5 \pm 1 \mathrm{fm} / c$. The impact of replacing a first-order by a 1QCD EoS is illustrated in Fig. 3 right. In the LMR, the QGP yield increases at the expense of the hadronic one, resulting in a very similar total. However, at intermediate mass, larger QGP temperatures resulting from the nonperturbative reduction of the lattice EoS increase the total yield significantly, which is favored by the data.

Dielectron measurements from the SPS and RHIC are summarized in Fig. 4. The recent STAR data from the RHIC beam-energy scan [21] constitute a first systematic excitation function, establishing consistency with previous SPS results [20]. The strongly broadened $\rho$ spectral function plus a moderate QGP contribution, as found at the SPS, describe the data up to top RHIC energy. This indicates a universal emission source, with large contributions from around $T_{\mathrm{pc}}$ and hadronic medium effects driven by baryons and antibaryons. We recall, however, that these calculations cannot explain the large low-mass, low-momentum enhancement observed by PHENIX in central $\mathrm{Au}-\mathrm{Au}$, while the central STAR data tend to be slightly overestimated around $M \simeq 0.2 \mathrm{GeV}$. 

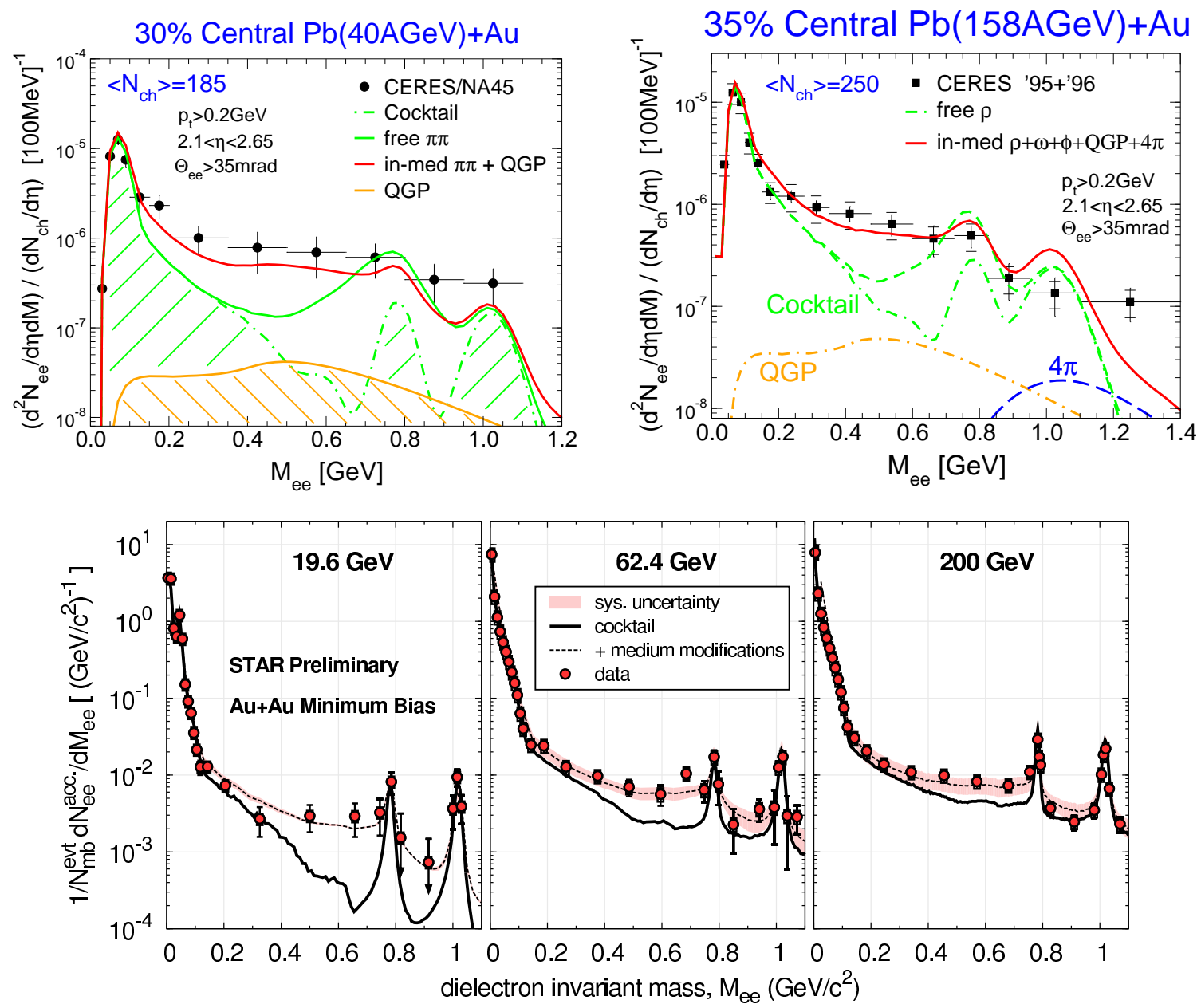

Figure 4: Excitation function of dielectron spectra from CERES/NA45 [20] at $\sqrt{s}=8.8 \mathrm{GeV}$ (upper left) and $17.3 \mathrm{GeV}$ (upper right) and from STAR at $\sqrt{s}=19.6,62.4$ and $200 \mathrm{GeV}$ (lower panels) [21].

\subsection{Direct Photons}

Direct photon radiation has been measured at RHIC and LHC and also shows a substantial excess over primordial and final-state hadron decay sources. The excess spectra carry inverse slopes of $T_{\text {eff }}=221 \pm 27 \mathrm{MeV}$ (RHIC) [22] and 301 $\pm 51 \mathrm{MeV}$ (LHC) [23], and an appreciable $v_{2}$. The latter is difficult to explain by early QGP radiation, but the inverse slopes actually point at "later" emission as well. Using the blue-shift expression, $T_{\mathrm{eff}} \simeq T \sqrt{(1+\beta) /(1-\beta)}$, with an average radial flow velocity of $\beta=0.3-0.4$, leads to emission temperatures of $T \simeq 160-200 \mathrm{MeV}$ and renders a large $v_{2}$ plausible. Nevertheless, it is not easily reproduced in realistic calculations.

In Ref. [24], LO QGP and in-medium hadronic rates have been evolved over a thermal fireball model. The latter has been constrained by measured hadron spectra and $v_{2}$, with a key feature of the bulk- $v_{2}$ leveling off around $T_{\mathrm{pc}}$, i.e., after ca. $5-6 \mathrm{fm} / c$ into the evolution of Au-Au at RHIC. This is not necessarily the case in hydrodynamic simulations [26, 27], but can be realized when utilizing a non-zero initial flow together with a rather compact initial energy density profile [25]. This, in particular, leads to the realization of "sequential freezeout", i.e., the kinetic decoupling of multi- 

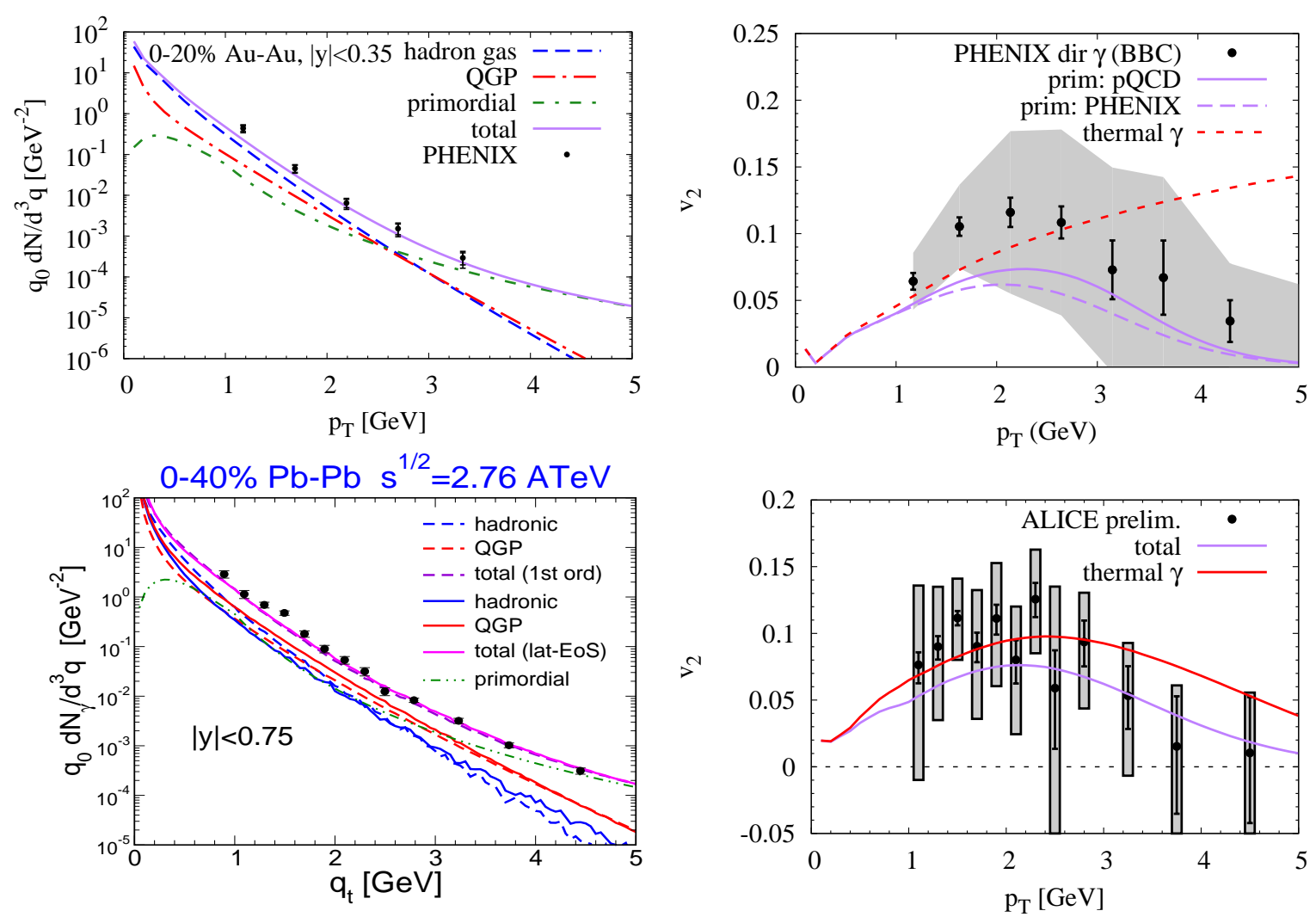

Figure 5: Direct photon spectra (left panels) and elliptic flow (right panels) in $0-20 \% \mathrm{Au}-\mathrm{Au}(\sqrt{s}=200 \mathrm{GeV}$ ) at RHIC (upper panels) and $0-40 \% \mathrm{PbPb}(\sqrt{s}=2.76 \mathrm{TeV}$ ) at LHC (lower panels).

strange hadrons $\left(\phi, \Xi, \Omega^{-}\right)$at chemical freezeout, $T_{\mathrm{ch}} \simeq T_{\mathrm{pc}}$. The resulting thermal photon spectra lead to approximate agreement with the PHENIX data, while the $v_{2}$ is at the lower end of the experimental uncertainty. At the LHC, the agreement with preliminary data in $0-40 \% \mathrm{~Pb}-\mathrm{Pb}(2.76 \mathrm{ATeV})$ is fair. The $q_{t}$ spectra illustrate a significant reshuffling of QGP and hadronic contributions when switching from first-order to $1 \mathrm{QCD}$ EoS, reiterating the large contributions from around $T_{\mathrm{pc}}$.

\section{Conclusions}

Electromagnetic radiation in heavy-ion collisions continues to illuminate the properties of the formed medium. Low-mass dilepton spectra and their interpretation are developing into a rather consistent picture, where the melting of the $\rho$ meson established at SPS seems to prevail also at RHIC. We have argued that this melting is theoretically compatible with chiral symmetry restoration and suggestive for a gradual change in the effective degrees of freedom in the system. Taken together with the temperature slopes extracted from the invariant-mass spectra, we may well have evidence for the long-sought for radiation from the QCD transition region. The inverse slopes and remarkable $v_{2}$ in the direct photons support this interpretation, even though a full theoretical understanding has not yet been achieved (possibly calling for additionally enhanced photon rates around $T_{\mathrm{pc}}$, and/or initial-state effects [29]). Clearly, a dilepton $v_{2}$ measurement, as well as precision mass spectra at collider energies, are needed to further test and deepen our understanding. 


\section{Acknowledgment}

This work has been supported by the U.S. National Science Foundation under grants no. PHY0969394 and PHY-1306359, and by the A.-v.-Humboldt Foundation.

\section{References}

[1] R. Rapp, J. Wambach and H. van Hees, in Relativistic Heavy-Ion Physics, edited by R. Stock and Landolt Börnstein (Springer), New Series I/23A (2010) 4-1 [arXiv:0901.3289[hep-ph]].

[2] S. Leupold, V. Metag and U. Mosel, Int. J. Mod. Phys. E 19, 147 (2010).

[3] P.M. Hohler and R. Rapp, EPJ Web Conf. 36, 00012 (2012); in preparation (2013).

[4] C.A. Dominguez and M. Loewe, Phys. Lett. B 233, 201 (1989).

[5] S. Weinberg, Phys. Rev. Lett. 18, 507 (1967).

[6] T. Das, V.S. Mathur, and S. Okubo, Phys. Rev. Lett. 19, 859 (1967).

[7] J.I. Kapusta and E.V. Shuryak, Phys. Rev. D 49, 4694 (1994).

[8] P.M. Hohler and R. Rapp, Nucl. Phys. A 892, 58 (2012).

[9] P. Gerber and H. Leutwyler, Nucl. Phys. B 321, 387 (1989).

[10] I. Jameson, A.W. Thomas and G. Chanfray, J. Phys. G 18, L159 (1992).

[11] M.C. Birse and J.A. McGovern, Phys. Lett. B292, 242 (1992).

[12] E. Braaten, R.D. Pisarski, and T.-C. Yuan, Phys. Rev. Lett. 64, 2242 (1990).

[13] H.T. Ding et al., Phys. Rev. D 83, 034504 (2011).

[14] S. Borsanyi et al. [Wuppertal-Budapest Coll.], JHEP 1009, 073 (2010).

[15] B.B. Brandt, A. Francis, H.B. Meyer and H. Wittig, JHEP 1303, 100 (2013).

[16] R. Rapp, arXiv:1304.2309 [hep-ph].

[17] S. Turbide, R. Rapp and C. Gale, Phys. Rev. C 69, 014903 (2004).

[18] P.B. Arnold, G.D. Moore and L.G. Yaffe, JHEP 0112, 009 (2001).

[19] R. Arnaldi et al. [NA60 Coll.], Phys. Rev. Lett. 96, 162302 (2006); Eur. Phys. J. C 61, 711 (2009).

[20] G. Agakichiev et al. [NA45 Coll.], Eur. Phys. J. C41, 475 (2005); Phys. Rev. Lett. 91, 042301 (2003).

[21] F. Geurts et al. [STAR Coll.], Nucl. Phys. A904-905 2013, 217c (2013).

[22] A. Adare et al. [PHENIX Coll.], Phys. Rev. Lett. 104, 132301 (2010); ibid. 109, 122302 (2012).

[23] M. Wilde et al. [ALICE Coll.], Nucl. Phys. A904-905, 573c (2013); arXiv:1212.3995 [hep-ex].

[24] H. van Hees, C. Gale and R. Rapp, Phys. Rev. C 84 (2011) 054906.

[25] M. He, R.J. Fries and R. Rapp, Phys. Rev. C 85, 044911 (2012).

[26] R. Chatterjee, D.K. Srivastava and U. Heinz, arXiv:0901.3270 [nucl-th].

[27] M. Dion et al., Phys. Rev. C 84, 064901 (2011).

[28] H. van Hees et al., in preparation (2013).

[29] A. Bzdak and V. Skokov, Phys. Rev. Lett. 110, 192301 (2013). 